\title{
Setup errors in patients with head-neck cancer (HNC), treated using the Intensity Modulated Radiation Therapy (IMRT) technique: how it influences the customised immobilisation systems, patient's pain and anxiety
}

Massimiliano Contesini ${ }^{1}$, Monica Guberti ${ }^{*}$, Roberta Saccani ${ }^{3}$, Luca Braglia ${ }^{4}$, Cinzia lotti ${ }^{3}$, Andrea Botti ${ }^{5}$, Emilio Abbati ${ }^{3}$ and Marina lemmi ${ }^{2}$

\begin{abstract}
Background: In patients with head-neck cancer treated with IMRT, immobility of the upper part of the body during radiation is maintained by means of customised immobilisation devices.

The main purpose of this study was to determine how the procedures for preparation of customised immobilisation systems and the patients characteristics influence the extent of setup errors.

Methods: A longitudinal, prospective study involving 29 patients treated with IMRT. Data were collected before CT simulation and during all the treatment sessions (528 setup errors analysed overall); the correlation with possible risk factors for setup errors was explored using a linear mixed model.

Results: Setup errors were not influenced by the patient's anxiety and pain. Temporary removal of the thermoplastic mask before carrying out the $\mathrm{CT}$ simulation shows statistically borderline, clinically relevant, increase of setup errors (+24.7\%, 95\% Cl: $-0.5 \%-55.8 \%)$. Moreover, a unit increase of radiation therapists who model the customised thermoplastic mask is associated to a $-18 \%(-29.2 \%--4.9 \%)$ reduction of the errors.

The setup error is influenced by the patient's physical features; in particular, it increases both in patients in whom the treatment position is obtained with 'Shoulder down' (+27.9\%, 2.2\% - 59.7\%) and in patients with 'Scoliosis/kyphosis' problems (+65.4\%, 2.3\% - 164.2\%). Using a 'Small size standard plus customized neck support device' is associated to a $-52.3 \%(-73.7 \%--11.2 \%)$ reduction.

The increase in number of radiation therapists encountered during the entire treatment cycle does not show associations. Increase in the body mass index is associated with a slight reduction in setup error by $(-2.8 \%,-5 \%--0.7 \%)$.

Conclusion: The position of the patient obtained by forcing the shoulders downwards, clinically significant scoliosis or kyphosis and the reduction of the number of radiation therapists who model the thermoplastic mask are found to be statistically significant risk factors that can cause an increase in setup errors, while the use of 'Small size' neck support device and patient BMI can diminish them.
\end{abstract}

Keywords: HNC, IMRT, Setup error, Pain, Anxiety, Customised immobilisation systems

\footnotetext{
* Correspondence: monica.guberti@asmn.re.it

${ }^{2}$ Nursing Directorate, Arcispedale Santa Maria Nuova-IRCCS, Reggio Emilia, Italy

Full list of author information is available at the end of the article
} 


\section{Introduction}

Intensity modulated radiation therapy is the standard for the treatment of head-neck cancer [1], because it allows a distribution in high doses in conformity to the tumour, saving the adjacent normal structures.

IMRT generates high gradient doses on the target, with rapid drop in the latter to the level of normal structures; as a result, this technique requires extremely high precision in treatment; in fact, very minor positioning errors can affect the target cover and increase the dose to organs at risk (OARs) [2, 3].

Image guided radiation therapy (IGRT) techniques have been developed and used to ensure accurate positioning of the interfraction patient and obtain a repeatable dispensing of the dose [4-7].

In patients with head-neck cancer (HNC) treated with IMRT, immobility of the upper part of the body during radiation is usually maintained by means of customised immobilisation devices such as head-neck-shoulder thermoplastic masks $[8,9]$. The main purpose of this study was to explore how patients characteristics, the procedures for preparation of customised immobilisation systems and their subsequent use can influence the accuracy and reproducibility of treatment.

\section{Methods}

\section{Study design and assessment procedure}

This is a longitudinal, prospective study: data were collected before CT simulation and during all the treatment sessions. All the patients enrolled were identified by a numeric code to allow blind data analysis and ensure the patient's privacy. Written informed consent was obtained during the first radiation therapy visit. The information was provided by the same radiation therapists for all the patients involved in the study, in order to ensure homogeneity. The local Ethics Committee approved the study.

\section{Setting}

In our department radiation therapy treatments can be performed using the TomoTherapy ${ }^{\oplus} \mathrm{Hi}$-Art ${ }^{\oplus}$ System 3.0 (Accuray Incorporated, Madison, WI), which involves a helical fan beam scan using 3.5MV photons and an arcshaped xenon CT detector array mounted on the opposite side of the ring gantry Mega Volt Fan Beam Computed Tomography (MVFBCT).

All patients with head-neck tumour were immobilised using a 5-points mask: four fixing points for the neck and shoulders plus an additional one on the head (Acquaplast $\mathrm{RT}^{\ominus}$ Fibreplast ${ }^{\mathrm{r} M}$ Thermoplastics, Avondale, PA, USA). The patient's position was maintained by means of a standard rigid base made of carbon fibre, created specially to stabilise the patient's head and neck during IMRT treatments. This supporting base of the patient is always fixed to the Computer Tomography and the Tomotherapy beds.

To maintain the patient's position standard neck supports were used in different sizes (Large, Small size), connected to the base. In some cases, to improve patient comfort, an additional customised neck support was used (MOLDCARE ${ }^{\oplus}$ CUSHION For Head, Neck and Small Regions Rt -4492S, ALCARE Co., Ltd. Tokyo, Japan). All the patients enrolled were subjected to Virtual Simulation Computed Tomography (GE Medical System HiSPEED $\mathrm{NX} / \mathrm{I})$ using the same technical protocol $(120 \mathrm{kV}, 250 \mathrm{~mA}$, Thickness of acquisition $3 \mathrm{~mm}$, Pitch 3, Thickness of reconstruction $3 \mathrm{~mm}$, Reconstruction interval $3 \mathrm{~mm}$ ).

Before each treatment, patient positioning was obtained by means of alignment of the lasers of the room with 4 reference points positioned on the "mask" at the time of CT simulation.

In IGRT-IMRT treatments dispensed in Tomotherapy, the MVFBCT images were acquired daily before each treatment and matched with those obtained during the CT simulation. Alignment of the images was obtained by means of automatic software tools and, if necessary, also with manual adjustments [10-12].

We considered the translational mediolateral (ML), craniocaudal (CC), and anteroposterior (AP) deviations; antero-posterior correction of $3.8 \mathrm{~mm}$ is applied for the systematic AP setup error caused by couch sag [13-16] that occurs when the patient is moved to the treatment isocenter (inside the bore) from virtual isocenter located $70 \mathrm{~cm}$ outside. Rotational corrections like pitch, roll and yaw were not recorded.

\section{Patients}

We prospectively collected data for a sample of consecutive patients with head and neck cancer, treated with the IMRT technique by our department from September 2011 to August 2013.

The eligibility criteria were: female and male patients with head and neck cancer who underwent intensity modulated radiation therapy, $\geq 14$ years old and $\leq 85$ years old, without cognitive disfunctions, performance status score of 0-1 [17] and with written informed consent.

\section{Data collection}

For all the patients, the thermoplastic mask and other custom immobilization devices were prepared before CT simulation, on the basis of the same internal operating protocol.

We created an ad-hoc report to record all the environmental and technical variables that might have a correlation with the setup error. On the baseline (BL) day of CT simulation we recorded: anxiety [18] and pain [19-24], before starting the simulation procedure (more on the measurements scales below); patient's weight and height [25, 26]; intent of radiation therapy; variable influencing patient 
positioning such as shoulders forced down, scoliosis or kyphosis; some patient and environment variables such as presence of mobile dental prosthesis, use of bite for locking of the jaw, tracheostomy, voluminous beard, voluminous hair, room temperature of the CT room and temperature of the water used for shaping the thermoplastic mask; type of standard neck supports used and the eventual addition of customized neck support; number of radiation therapy team members who worked together to shape the thermoplastic mask and temporary removal of the mask before the acquisition of CT simulation. On the first day of every week of treatment we measured the patient's weight. Finally on each treatment day we recorded: Mediolateral (ML), craniocaudal $(\mathrm{CC})$, and anteroposterior (AP) deviations; radiation therapists executing the procedure; anxiety and pain, before starting the treatment session; changes related to the presence of mobile dental prosthesis, use of dental bite, tracheostomy, beard or voluminous hair, temperature of the treatment room.

The anxiety was measured using the STAI Y form, a test validated worldwide [27] consisting of two scales: the TRATTO-A scale, which measures the predisposition for anxiety, and the STATE-A scale which evaluates the anxiety state, i.e. the emotional state at a given moment. Only STATE-A was used in the study, which consists of 20 items; the compilation, done directly by the patient, requires an average of 5-7 min. The test was administered to the patient in the waiting room.

Pain was measured using the Numeric Pain Rating Scale, [19-24] a numeric scale that describes the intensity of pain, ranging from 0 (no pain) to 10 (max value of pain); the measurement was performed directly by the patients themselves in the waiting rooms.

\section{Study size}

In absence of a-priori hypothesis given the exploratory nature of the study, no formal sample sizing was performed. Nonetheless, a limited measurement period (recruiting: September 2011 to August 2013, data collection: first 6 weeks of treatment for each patient) was defined, in order to ensure general feasibility, homogeneity of the procedures and of the data collected.

\section{Statistical methods}

Clinical and demographic data were expressed in terms of frequency and percentage for categorical variables, mean \pm standard deviation for symmetric quantitative variables, median $+\mathrm{IQR}$ for skewed ones.

Overall mean error $(M)$, systematic SD $(\Sigma)$ and random SD $(\sigma)$ were calculated, both for full sample and by groups (determined according to site) of patients.

In order to explore the correlation between relevant variable and treatment setup errors, we adopted a single Euclidean distance measure. Setup error (in any of the three directions) can assume positive or negative values; regardless of the direction, the more the error deviates from value 0 the more unfavourable the it is. Therefore, to collapse all the three errors (cranial-caudal, mediallateral and anterior-posterior) in one single measurement readily available for multivariate analysis, we considered each setup error as a vector and calculated 3dimension modulus as an overall measure of error severity; accordingly, total error $e_{t o t}$ was simply calculated as Euclidean distance, as follows:

$$
e_{t o t}=\sqrt{e_{m l}^{2}+e_{c c}^{2}+e_{a p}^{2}}
$$

where $e_{m l}$ is medial-lateral (ML), $e_{c c}$ is cranial-caudal (CC) and $e_{a p}$ is anterior-posterior (AP) error (to simplify notation we avoided patient and time subscripting; however $e_{t o t}$ has to be considered as overall setup error for patient $i$ at time $t$ ). The total error, expressed in $\mathrm{mm}$, was log-transformed (in order to mitigate the positiveskewness effects on the residuals normality) and then analysed using a linear mixed effects model (completecase analysis). This method was choosed to accommodate non-independency of the observations sampled (eg. due to clustering within individuals): the method assumes that setup errors can be explained in terms of both fixed and random effects. Fixed effects represent the effects of factors of intrinsic interest (in our case those that can be associated to setup error) because of being repeatable in other populations (eg number of therapists, mask removal etc.); random effects represent random deviation (from the relation depicted by the fixed effects) associated to factors of no intrinsic interest (not strictly repeatable, eg the single patient) which are nonetheless considered in the estimation process [28].

Covariates used in the model were suggested by clinical/ technical interest and feasibility (some patient characteristic, eg voluminous beard etc., were highly imbalanced and therefore were ignored for the correlation analysis): fixed effect variables were shoulders down, scoliosis/kyphosis and mask removal (No/Yes variables), type of neck support device (categorical variable), number of radiation therapists involved at baseline, pain, anxiety, number of radiation therapists encountered during the treatment weeks and body mass index (continuous variables treated as such also in the statistical model); a random patient intercept complete the model presented. We compared this model with one including some additional variables (fixed effects: pain and anxiety before each treatment session, both hair and temperature at baseline and before each treatment session; random intercept: treatment technician), concluding that these latter were not needed $(P=0.98)$.

The base group for the model reported is represented by patients without 'Shoulders down', 'Scoliosis/kyphosis' and 'Mask removal' at baseline setup; with 'Large size 
standard plus customized neck support device' (the most common); followed by 2 radiation therapists at baseline for mask and setup (the minimum/standard encountered); with no pain (pain $=0$ ); with mid-point anxiety $($ anxiety $=50)$; patient who during the entire treatment cycle, was followed by 12 radiation therapists (minimum) during treatment weeks; with a mean BMI.

In order to ease interpretation of results, coefficients (and their confidence intervals) were transformed using $\left(\left((\exp (\text { coefficient })-1)^{*} 100\right)-100\right)$ and presented using a forestplot-like graph: this leads to an estimate of each factor contribution as percentage variation (compared to an otherwise identical patients without it).

Confidence intervals (considering a 0.95 confidence level) and models comparison were performed with bootstrap methods [29]; a confidence interval not including the threshold of no differences ( $0 \%$ for transformed coefficients) denoted the contribution of the factor analysed as statistically significant. Statistical analysis was carried out using R 3.3.2.

\section{Results}

\section{Patients}

The number of eligible patients screened at our radiation therapy centre for IMRT treatment from September 2011 to August 2013 was thirty-nine (39); seven (7) patients did not give their consent for research; two (2) patients were discontinued from the study since their treatment had to be re-planned and the mask had to be remade as it had lost its accuracy during the radiation therapy cycle; one patient (1) dropped out because of increased pain and poor collaboration. Therefore the analysed sample consisted of twenty-nine (29) consecutive patients (for a total of 528 daily images); descriptive statistics of the patients at baseline (CT Simulation) are shown in (Table 1).

\section{Outcome descriptives}

Overall mean error $(M)$, systematic error $(\Sigma)$ and random error $(\sigma)$ are shown in (Table 2).

The mean displacements in $\mathrm{ML}, \mathrm{CC}$ and $\mathrm{AP}$ were $-0.4,2.0$ and $0.8 \mathrm{~mm}$. Systematic and random errors were 3.1, 1.6, 1.3 and 2.2, 1.7, 1.7 in the ML, CC and AP directions, respectively.

\section{Main results}

The mixed effects model is presented in (Table 3) while a graphical summary of results is plotted in (Fig. 1).

Considering the graph, factors associated with a diminished setup error have a confidence interval on the left of the vertical line (negative percent variation), while others with confidence interval on the right are associated to an increased setup error: the percentage

Table 1 Baseline descriptive statistics (CT simulation)

\begin{tabular}{llll}
\hline Patients, n \% & & 29 & 100 \\
& & & \\
& Female & 9 & $31.0 \%$ \\
& Male & 20 & $69.0 \%$ \\
Age, Median (IQR) & & 65.7 & $(58.9-71.9$ \\
Site, n\% & & \\
& Nasopharynx & 5 & $17.2 \%$ \\
& Oropharynx & 13 & $44.8 \%$ \\
& Hypopharynx & 5 & $17.2 \%$ \\
& Oral cavity & 4 & $13.8 \%$ \\
& Other & 2 & $6.9 \%$
\end{tabular}

Setting, n \%

\section{Radical}

Post surgery

Shoulders down, n \%

No

Yes

Not available

Scoliosis/kyphosis, n \%

No

$\mathrm{BMI}$, mean (sd)

Mask removal, n \%

$\begin{array}{lll}\text { No } & 13 & 44.8 \% \\ \text { Yes } & 15 & 51.7 \% \\ \text { Not available } & 1 & 3.4 \%\end{array}$

Neck support device, $n \%$

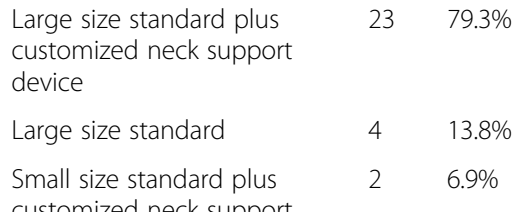

Baseline radiation therapists, n \%

\section{$23 \quad 79.3 \%$ \\ $6 \quad 20.7 \%$}

\section{$18 \quad 62.1 \%$ \\ $10 \quad 34.5 \%$ \\ $13.4 \%$}

$26 \quad 89.7 \%$

$3 \quad 10.3 \%$

$24.07 \quad(4.34)$

customized neck support device

\begin{tabular}{llll} 
& 1 & 2 & $6.9 \%$ \\
& 2 & 23 & $79.3 \%$ \\
& 3 & 3 & $10.3 \%$ \\
Pain, Median (IQR) & 4 & 1 & $3.4 \%$ \\
Anxiety, Median (IQR) & & 0 & $(0-2)$ \\
\hline
\end{tabular}

variation is estimated by referencing to a patient identical in every aspect but the risk factor considered in turn.

Having 'Shoulders down' and 'Scoliosis/kyphosis' is associated to a $27.9 \%$ (95\% CI: $2.2 \%-59.7 \%$ ) and $65.4 \%$ 
Table 2 Population setup errors

\begin{tabular}{|c|c|c|c|c|}
\hline & & Tome & & \\
\hline & & $\bar{M}$ & $\Sigma$ & $\sigma$ \\
\hline All patients & & & & \\
\hline & Mediolateral & -0.4 & 3.1 & 2.2 \\
\hline & Craniocaudal & 2.0 & 1.6 & 1.7 \\
\hline & Anteroposterior ${ }^{a}$ & 0.8 & 1.3 & 1.7 \\
\hline Main sites: & & & & \\
\hline Nasophary & & & & \\
\hline & Mediolateral & 1.5 & 2.8 & 2.6 \\
\hline & Craniocaudal & 1.2 & 1.1 & 2.5 \\
\hline & Anteroposterior ${ }^{a}$ & 0.7 & 1.8 & 1.5 \\
\hline Oropharyn & & & & \\
\hline & Mediolateral & -1.6 & 2.9 & 2.5 \\
\hline & Craniocaudal & 1.8 & 1.4 & 1.6 \\
\hline & Anteroposterior ${ }^{a}$ & 0.7 & 1.4 & 1.9 \\
\hline Hypophary & & & & \\
\hline & Mediolateral & -0.3 & 3.4 & 1.7 \\
\hline & Craniocaudal & 2.6 & 1.7 & 1.6 \\
\hline & Anteroposterior ${ }^{a}$ & 1.1 & 0.9 & 1.3 \\
\hline Oral cavity & & & & \\
\hline & Mediolateral & 1.2 & 1.5 & 1.4 \\
\hline & Craniocaudal & 2.0 & 1.4 & 1.3 \\
\hline & Anteroposterior ${ }^{a}$ & 0.5 & 1.2 & 1.6 \\
\hline Other & & & & \\
\hline & Mediolateral & 2.0 & 3.3 & 1.9 \\
\hline & Craniocaudal & 4.6 & 3.0 & 1.5 \\
\hline & Anteroposterior ${ }^{a}$ & 1.7 & 1.5 & 1.3 \\
\hline
\end{tabular}

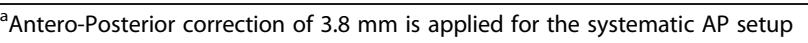
error caused by couch sag

Table 3 A linear mixed effect model: fixed effect coefficients

\begin{tabular}{|c|c|c|c|c|}
\hline & \multirow[t]{2}{*}{ Estimate } & \multirow{2}{*}{$\begin{array}{l}\text { Std. } \\
\text { Error }\end{array}$} & \multicolumn{2}{|l|}{$95 \% \mathrm{Cl}$} \\
\hline & & & Lower limit & Upper limit \\
\hline Intercept & 1.327 & 0.099 & 1.137 & 1.518 \\
\hline Shoulders down (BL) $\left(^{*}\right)$ & 0.246 & 0.115 & 0.022 & 0.468 \\
\hline Scoliosis/kyphosis (BL) & 0.503 & 0.244 & 0.023 & 0.972 \\
\hline Mask removal (BL) & 0.221 & 0.114 & -0.005 & 0.444 \\
\hline \multicolumn{5}{|l|}{ Neck support device (BL): } \\
\hline Large size, standard & 0.130 & 0.185 & -0.240 & 0.491 \\
\hline Small size, custom & -0.740 & 0.309 & -1.337 & -0.119 \\
\hline Baseline technicians (BL) & -0.198 & 0.074 & -0.346 & -0.051 \\
\hline Pain (BL) & -0.027 & 0.042 & -0.110 & 0.055 \\
\hline Anxiety (BL) & -0.005 & 0.005 & -0.016 & 0.005 \\
\hline Treament technicians & -0.043 & 0.045 & -0.132 & 0.045 \\
\hline Body mass index & -0.029 & 0.011 & -0.051 & -0.007 \\
\hline
\end{tabular}

(*) 'BL' means a baseline measured variable
$(2.3 \%-164.2 \%)$ increase in mean setup error respectively. Mask removal is associated to a (borderline) non significant increase in setup errors $(+24.7 \%,-0.5 \%-$ $55.8 \%$ ). Using a 'Small size standard plus customized neck support device' is associated with a $-52.3 \%$ $(-73.7 \%,-11.2 \%)$ setup error contraction; less clear indications are available on the use of customized neck support (base group) in patients with 'Large size standard' neck support device.

No significant relations were found between the number of radiation therapists encountered during the entire treatment schedule and setup errors; on the other hand, a unit increase in the number of radiation therapists involved in CT Simulation for making the mask is associated with a $-18 \%(-29.2 \%--4.9 \%)$ decrease of setup errors.

Increased BMI coefficient suggests a mild protective effect, $-2.8 \%(-5 \%,-0.7 \%)$ per unit; ., no associations were found for anxiety and pain.

\section{Discussion}

\section{Key results and limitations}

This study was a mono-institutional experience, conducted in an explorative way without a formal process of study dimensioning, aimed at exploring the correlation between setup error and possible risk factors. First, statistically significant results were obtained for Scoliosis/ kyphosis; shoulders down was, as expected, predictive of clinically relevant increase of set-up error. These results may be explained by the fact that such anatomical conditions of the patient are associated with less comfort and result in lower reproducibility of the position. The use of a 'Small size standard plus customized neck support device' is associated with reduction in set-up errors; this is probably due to the fact that such an immobilization system ensures a greater restraint of the patient's head and neck.

The unit increase in the number of radiation therapists at baseline is associated with an overall reduction of average setup errors; this can be explained by the fact that increase in the number of radiation therapists involved in shaping the thermoplastic mask on the patient ensures greater accuracy in the preparation of the patient immobilisation system.

The results of the study show that temporary removal of the mask before CT simulation acquisition is associated with a clinically significant increases of the setup error, despite the association in not strictly statistical significant; this provides clues on the probable usefulness of immobilisation procedure in claustrophobic patients (in whom it is often necessary to remove the immobilisation system before the CT simulation).

The patient's pain and anxiety measured in the CT simulation phase were not associated with a clinically significant increase of setup errors. These results can be explained by the fact that the members of the radiation therapy team 


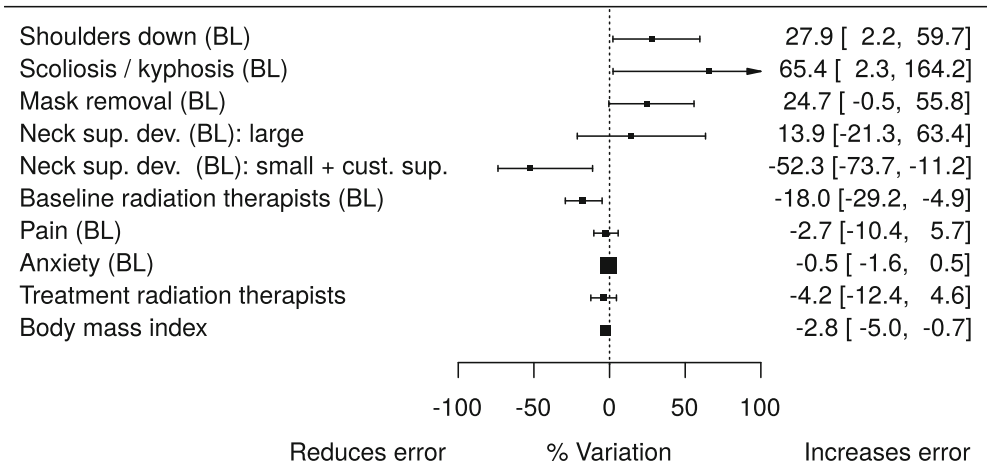

Fig. 1 Model results: setup error variation associated to each factor analysed

received training in communication strategies which may be used to reduce patient anxiety. Patient pain, despite increasing gradually during the radiation therapy cycle, was well controlled with pharmacological treatment.

The number of radiation therapists encountered during the treatment schedule was not associated with clinically relevant increase set-up error; this can be explained by the fact that the high standardization of treatment procedures ensures the reproducibility of the patient setup, which is not operator dependent. The results also showed that factors such as voluminous hair, room temperature and temperature of the water used for shaping the thermoplastic mask were not associated with SE.

To the best of our knowledge, this is the first study to prospectively investigate the relations between SE and the procedures for preparation of customized immobilization systems: however, these findings need to be confirmed. We can highlight as a point of weakness that geometric corrections such as pitch, roll, and yaw were not considered; moreover, we also need to report the limited numbers of patients.

\section{Conclusion}

Factor associated with an increased error were 'Shoulders down' and scoliosis/kyphosis; factor associated with a diminished error were the use of small size plus custom neck support device, the number of radiation therapists involved in shaping the thermoplastic mask during CT Simulation and BMI.

The results of the study were obtained from a small sample size and need to be confirmed, but provide suggestions useful to the radiation therapist in the treatment preparation phase and in the positioning of the patient daily during therapy.

\section{Abbreviations}

AP: Anteroposterior; BMI: Body mass index; CC: Craniocaudal; DRRs: Digitally reconstructed radiography; EPID: Electronic Portal Imaging Device; HNC: Headneck cancer; IGRT: Image guided radiation therapy; IMRT: Intensity modulated radiation therapy; ML: mediolateral; MVFBCT: Mega Volt Fan Beam Computed Tomography; OARs: Organs at risk; SE: Setup errors

\section{Acknowledgements}

This research was developed with the support of the following radiation therapists working in the Department of Oncology and Advanced Technologies of the Hospital of Reggio Emilia: N. Moretti, D. Bertoni, F. Faedda, C. Mercati, L. Casotti, C. Monteduro, F.M. Tambasco, E. Tedeschi, G. Tagliavini, A. Ferrarrini, R. Raffaelli, A. Sirri, G. Falchi, C. Daolio, D. Franchi, A. Quarta, L. Balducci, G. Di Natali, C. Lopes, B. Scazzina.

Funding

No funding was received.

\section{Availability of data and materials}

The dataset generated during the current study is not publicly available because it contains clinical as well as demographic data. However, selected data can be requested from the corresponding author on reasonable request.

\section{Authors' contributions}

$M C, E A, R S, M I$ and $M G$ conceived the study. MC, EA and MG designed the study, collected and analysed the data and prepared the manuscript. LB made the statistical analysis and revised the manuscript. $C l$ and $A B$ gave important intellectual input and revised the manuscript. All authors approved the final version for submission.

\section{Competing interests}

The authors declare that they have no competing interests.

\section{Consent for publication}

All patients treated at the Radiation Oncology Department, Arcispedale S.Maria Nuova -IRCCS, Reggio Emilia, Italy, gave their informed consent on the scientific use and publication of their anonymized data.

\section{Ethics approval and consent to participate}

The present work is based on a prospective analysis of clinical data. All patients gave their informed consent that clinical data can be used for scientific analysis and scientific pubblication. The project was approved by the local Ethics Committee at Reggio Emilia (n² 201100145 75-20 November 2011).

\section{Publisher's Note}

Springer Nature remains neutral with regard to jurisdictional claims in published maps and institutional affiliations.

\section{Author details}

${ }^{1}$ Human Resource Development - Training, Azienda Unità Sanitaria Locale, Reggio Emilia, Italy. ${ }^{2}$ Nursing Directorate, Arcispedale Santa Maria Nuova-IRCCS, Reggio Emilia, Italy. ${ }^{3}$ Radiation Oncology Department, Arcispedale S. Maria Nuova -IRCCS, Reggio Emilia, Italy. ${ }^{4}$ Scientific Directorate, Arcispedale S. Maria Nuova - IRCCS, Reggio Emilia, Italy. ${ }^{5}$ Medical Physics Department, Arcispedale S.Maria Nuova -IRCCS, Reggio Emilia, Italy. 
Received: 15 November 2016 Accepted: 13 April 2017 Published online: 27 April 2017

\section{References}

1. Hong TS, Tomé WA, Harari PM. Intensity-modulated radiation therapy in the management of head and neck cancer. Curr Opin Oncol. 2005;17:231-5.

2. Manning MA, Wu Q, Cardinale RM, et al. The effect of setup uncertainty on normal tissue sparing with IMRT for head-and neck cancer. Int J Radiation Oncology Biol Phys. 2001;51:1400-9.

3. Samuelsson A, Mercke C, Johansson KA. Systematic set-up errors for IMRT in the head and neck region: effect on dose distribution. Radiother Oncol. 2003;66:303-11.

4. Hurkmans CW, Remeijer P, Lebesque JV, Mijnheer BJ. Set- up verification using portal imaging; review of current clinical practice. Radiother Oncol. 2001;58:105-20.

5. Cui Y, Galvin JM, Straube WL, et al. Multi-system verification of registrations for image-guided radiotherapy in clinical trials. Int J Radiation Oncology Biol Phys. 2011:81:305-12

6. Sharon Qi X, Hu Angie $Y$, Lee Steve $P$, et al. Assessment of Interfraction Patient Setup for Head-and-Neck Cancer Intensity Modulated Radiation Therapy Using Multiple Computed Tomography-Based Image Guidance. Int J Radiation Oncology Biol Phys. 2013:86:432-9.

7. Djordjevic M, Sjöholm E, Tellgren O, Sorgini B. Assessment of residual setup errors for anatomical sub-structures in image-guided head-and-neck cancer radiotherapy. Acta Oncol. 2014;53:646-53.

8. Sharp L, Lewin F, Johanssom H, Payne D, Gerhardsson A, Rutqvist LE. Randomized trial on two types of thermoplastic masks for patient immobilization during radiation therapy for head-and-neck cancer. Int J Radiation Oncology Biol Phys. 2005;61:250-6.

9. Bentel GC, Marks LB, Hendren K, Brizel DM. Comparison of two head and neck immobilization systems. Int J Radiation Oncology Biol Phys. 1997:38:867-73.

10. Chatterjee S, Mott JH, Smyth G, Dickson S, Dobrowsky W, Kelly CG Clinical challenges in the implementation of tomotherapy service for head and neck cancer patients in a regional UK radiotherapy centre. $\mathrm{Br} J$ Radiol. 2011;81:358-66.

11. Graff P, Kirby N, Weinberg V, et al. The Residual Setup Errors of Different IGRT Alignment Procedures for Head and Neck IMRT and the Resulting Dosimetric Impact. Int J Radiation Oncology Biol Phys. 2013;86:170-6.

12. Chen AM, Farwell DG, Luu Q, Donald PJ, Perks J, Purdy JA. Evaluation of the Planning Target Volume in the Treatment of Head and Neck Cancer with Intensity-Modulated Radiotherapy: What Is the Appropriate Expansion Margin in the Setting of Daily Image Guidance? Int J Radiation Oncology Biol Phys. 2010;81:943-9.

13. Shubert L, Westerly $\mathrm{D}$, Tomé W, et al. A comprehensive assessment by tumor site of patient setup using daily MVCT imagaing from more than 3800 helical tomotherapy treatments. Int J Radiat Oncol Biol Phys. 2009; 73(4):1260-9.

14. Houghton F, Benson RJ, Tudor GSJ, et al. An assessment of action levels in imaging strategies in Head and Neck cancer using tomotherapy. Are our margins adequate in the absence of image guidance? Clin Oncol. 2009; 21(9):720-7.

15. Smith $S$, Yartsev S, Van Dyk J. Evaluation of megavoltage $C T$ imaging protocol in patients with lung cancer. J Med Imaging Radiat Oncol. 2010;54(1):62-8.

16. Yeung TPC, Yartsev S, Rodrigues G, Bauman G. Evaluation of image-guide strategies with helical tomotherapy for localised prostate cancer. J Med Imaging Radiat Oncol. 2011;55(2):220-8.

17. Oken MM, Creech RH, Tormey DC, et al. Toxicity and response criteria of the Eastern Cooperative Oncology Group. Am J Clin Oncol. 1982;5:649-55.

18. Jensen MP, Turner JA, Romano JM, Fisher LD. Comparative reliability and validity of chronic pain intensity measures. Pain. 1999;83:157-62.

19. Hoggart WB. Pain: a review of three commonly used pain rating scales. J Clin Nurs. 2005;14:798-804.

20. Turk DC, Melzack R. Handbook of Pain Assessment, Third Edition. NY: Guilford Presse; 2011

21. Shannon MM, Ryan MA, D'Agostino N, Brescia FJ. Pain Assessment in Advanced Cancer. J Pain Symptom Manag. 1995;10:274-8.

22. van den Beuken-van Everdingen MHJ, de Rijke JM, Kessels AG, Schouten HC, van Kleef M, Patijn J. Prevalence of pain in patients with cancer: a systematic review of the past 40 years. Ann Oncol. 2007;18:1437-49.
23. Downie WW, Leatham PA, Rhind PA, et al. Studies with pain rating scales. Ann Rheum Dis. 1978:37:378-81.

24. De Bari B, Ait Erraisse M, Rabilloud M, et al. Does weight loss predict accuracy of setup in head and neck cancer patients treated with Intensity-Modulated Radiation Therapy? Radiol Med. 2012;117:885-91.

25. Liang Lai $Y$, Neng Yang S, An Liang J, et al. Impact of body-mass factors on setup displacement in patients with head and neck cancer treated with radiotherapy using daily on-line image guidance. Radiat Oncol. 2014;9:19. doi:10.1186/1748-717X-9-19.

26. Pehlivan $B$, Pichenot $C$, Castaing $M$, et al. Interfractional set-up errors evaluation by daily electronic portal imaging of IMRT in head and neck cancer patients. Acta Oncol. 2009;48:440-5.

27. Spielberger CD. State-Trait Anxiety Inventory (Form Y). Manual. Palo Alto: Mind Garden; 1983. p. 9-23.

28. West B, Welch K, Galecki A. Linear Mixed Models. A Practical Guide Using Statistical Software. $2^{\text {nd }}$ ed. CRC press, Boca Raton, Florida; 2014

29. r-sig-mixed-models FAQ http://glmm.wikidot.com/faq. Access ed 8 Mar 2017

\section{Submit your next manuscript to BioMed Central and we will help you at every step:}

- We accept pre-submission inquiries

- Our selector tool helps you to find the most relevant journal

- We provide round the clock customer support

- Convenient online submission

- Thorough peer review

- Inclusion in PubMed and all major indexing services

- Maximum visibility for your research

Submit your manuscript at www.biomedcentral.com/submit 